Supp Info: Isoprene Exposure in the United States

\title{
Supp Info: Isoprene Exposure in the United States based on Urinary IPM3: NHANES 2015-2016
}

\author{
Chloe Biren, Luyu Zhang, Deepak Bhandari, ${ }^{*}$ Benjamin C. Blount, and Víctor R. De Jesús
} Centers for Disease Control and Prevention Division of Laboratory Sciences, Tobacco and Volatiles Branch Atlanta, GA 30341

\section{Submitted for consideration for publication in Environ. Sci. Technol. Running Title: Isoprene Exposure in the United States}

\author{
*Corresponding Author: \\ Deepak Bhandari, $\mathrm{PhD}$ \\ Centers for Disease Control and Prevention \\ Division of Laboratory Sciences, Tobacco and Volatiles Branch \\ Email: xwo1@cdc.gov \\ Phone: 770-488-0939
}

Disclaimer: The findings and conclusions in this report are those of the authors and do not necessarily represent the official position of the Centers for Disease Control and Prevention.

\section{$\underline{\text { Highlights }}$}

- This paper is the first to evaluate urinary IPM3 as a biomarker of isoprene exposure in a largescale, population-representative study.

- We found that tobacco smoke is a major source of isoprene exposure in the US population based on urinary IPM3 concentrations in NHANES 2015-2016. 
Supp Info: Isoprene Exposure in the United States

This supplemental informations contains:

$41 \quad$ Number of pages: 4 (Page S1-S4)

42 Number of Tables: 3 (Tables S1-S3)

43 Number of Figures: 0

44

45 Table S1. USDA Food Codes and Logic for Apportioning Dietary Intake.

\begin{tabular}{|l|l|}
\hline Food Group & USDA Food Code and Additional Logic \\
\hline Milk and Milk Products & DR1IFDCD $=1$ \\
\hline Meat, Poultry, and Mixtures & DR1IFDCD $=2$ \\
\hline Eggs & DR1IFDCD $=3$ \\
\hline Legumes, Nuts, and Seeds & DR1IFDCD $=4$ \\
\hline Grain Products & DR1IFDCD $=5$ \\
\hline Fruits & DR1IFDCD $=6$ \\
\hline Vegetables & DR1IFDCD $=7$ \\
\hline Fats, Oils, and Salad Dressings & DR1IFDCD $=8$ \\
\hline Sugars, Sweets, and Beverages & DR1IFDCD $=9$ \\
\hline Coffee & $\begin{array}{l}\text { DR1IFDCD }=921 \\
\text { Then subtract total mass from DR1IFDCD } \\
=9\end{array}$ \\
\hline Beer and ales & $\begin{array}{l}\text { DR1IFDCD }=931 \\
\text { Then subtract total mass from DR1IFDCD } \\
=9\end{array}$ \\
\hline
\end{tabular}


Supp Info: Isoprene Exposure in the United States

64

65

66

67

68

69

Table S2. Sample-weighted Medians, Geometric Means, and $95^{\text {th }}$ Percentiles for Creatinine-adjusted Values $(\mu \mathrm{g} / \mathrm{g}$ creatinine) and Not Creatinine-adjusted Values $(\mathrm{ng} / \mathrm{mL})$ for Urinary IPM3 in NHANES $2015-2016(\mathrm{~N}=2,271) .^{\mathrm{a}}$

\begin{tabular}{|c|c|c|c|c|c|c|c|}
\hline \multirow{2}{*}{ Variable } & \multirow[b]{2}{*}{$\mathbf{N}$} & \multicolumn{3}{|c|}{ IPM3 ( $\mu \mathrm{g} / \mathrm{g}$ creatinine) } & \multicolumn{3}{|c|}{ IPM3 (ng/mL) } \\
\hline & & Median [25th, & GM $[95 \% C I]^{b}$ & 95th & Median [25th, 75th & GM $[95 \% \mathrm{CI}]^{\mathrm{b}}$ & 95th \\
\hline All & 2271 & $\overline{3} .39[2.13,6.12]$ & $4.27[3.91,4.62]$ & 53.6 & $2.96[1.55,6.38]$ & $3.66[3.27,4.04]$ & 46.6 \\
\hline \multicolumn{8}{|l|}{ Smoking Status } \\
\hline Exclusive Smoker ${ }^{\mathrm{c}}$ & 252 & $39.8[14.7,72.3]$ & $28.6[23.6,33.7]$ & 134 & $35.1[13.0,68.6]$ & $27.1[20.3,33.9]$ & 144 \\
\hline Non-user $^{\mathrm{d}}$ & 2019 & $3.05[2.03,4.93]$ & $3.23[3.07,3.39]$ & 10.7 & $2.62[1.32,4.95]$ & $2.73[2.58,2.88]$ & 13.4 \\
\hline Female & 1169 & $3.69[2.29,6.49]$ & $4.55[4.16,4.94]$ & 61.6 & $2.75[1.24,6.25]$ & $3.37[3.02,3.72]$ & 46.5 \\
\hline \multicolumn{8}{|l|}{ Age (yr) } \\
\hline $3-5$ & 257 & $6.06[3.99,8.86]$ & $6.25[5.46,7.03]$ & 17.7 & $2.46[0.850,4.45]$ & $2.50[2.14,2.87]$ & 12.0 \\
\hline $6-11$ & 261 & $4.11[2.59,6.49]$ & $4.30[3.84,4.76]$ & 13.8 & $2.68[1.32,5.70]$ & $2.88[2.61,3.15]$ & 14.3 \\
\hline $12-19$ & 323 & $2.44[1.78,3.79]$ & $2.68[2.46,2.91]$ & 8.68 & $2.51[1.23,4.85]$ & $2.78[2.45,3.12]$ & 13.9 \\
\hline \multicolumn{8}{|l|}{ Race/ethnicity } \\
\hline Non-Hispanic White & 686 & $3.53[2.30,6.45]$ & $4.61[4.00,5.22]$ & 65.8 & $2.79[1.63,6.21]$ & $3.71[3.14,4.27]$ & 54.6 \\
\hline Non-Hispanic Black & 453 & $2.99[1.88,7.01]$ & $4.07[3.48,4.66]$ & 37.9 & $4.09[2.04,10.5]$ & $5.12[4.28,5.96]$ & 65.3 \\
\hline Mexican-American & 468 & $3.11[2.05,5.65]$ & $3.62[3.38,3.86]$ & 22.5 & $3.03[1.44,6.05]$ & $3.26[2.97,3.55]$ & 25.9 \\
\hline Other Hispanic or other/multi race & 664 & $2.99[1.98,5.62]$ & $3.68[3.24,4.13]$ & 24.5 & $2.63[0.850,5.68]$ & $3.04[2.60,3.47]$ & 25.6 \\
\hline \multicolumn{8}{|l|}{ BMI } \\
\hline Healthy weight & 855 & $3.69[2.38,6.68]$ & $4.63[4.08,5.19]$ & 50.3 & $2.71[0.850,5.92]$ & $3.20[2.70,3.69]$ & 40.9 \\
\hline Underweight & 29 & $4.04[2.30,6.92]$ & $4.92[2.03,7.81]$ & 44.9 & $3.10[0.850,8.67]$ & $3.85[1.70,6.00]$ & 25.8 \\
\hline Overweight/Obese & 1387 & $3.21[2.04,5.85]$ & $4.09[3.69,4.49]$ & 53.7 & $3.02[1.75,6.53]$ & $3.90[3.45,4.34]$ & 54.3 \\
\hline
\end{tabular}

${ }^{\text {aSame data }}$ as in stratified serum cotinine regression models

$71{ }^{\mathrm{b}}$ Geometric mean [95\% confidence intervals]

72 cParticipants reporting using cigarettes (and no other tobacco products) 5 days prior to physical examination and with serum cotinine

73 measurement $>10 \mathrm{ng} / \mathrm{mL}$

74

dParticipants reporting not using cigarettes during 5 days prior to physical examination or with serum cotinine measurement $\leq 10 \mathrm{ng} / \mathrm{mL}$ 
Supp Info: Isoprene Exposure in the United States

83

84

85 Table S3. IPM3 Full model vs. Reduced model (omit CPD Group)

\begin{tabular}{|l|l|l|}
\hline & Variables & R-squared \\
\hline Full model & Creatinine (urine), CPD Group, Sex, Race/ethnicity, BMI, Food Group & 0.63 \\
\hline Reduced model & Creatinine (urine), Sex, Race/ethnicity, BMI, Food Group & 0.30 \\
\hline
\end{tabular}

86 